\title{
A seven-day high cocoa diet decreases oxidant and inflammatory properties of peritoneal macrophages in rats
}

\author{
S. Ramos-Romero, F. J. Pérez-Cano, T. Pérez-Berezo, C. Castellote, A. Franch and M. Castell \\ Department of Physiology, Institut de Recerca en Alimentació i Seguretat Alimentària, Faculty of Pharmacy, University of \\ Barcelona, Spain
}

Compared to traditional antioxidant foods, cocoa has a potent antioxidant capacity which leads it to be considered as a potentially beneficial ingredient. Previous in vitro studies have shown that a flavonoid-rich cocoa extract added to LPS-stimulated macrophages decreases the secretion of some cytokines and $\mathrm{NO}^{(1,2)}$, but there are no in vivo studies on the effects of cocoa intake on macrophages. The aim of the present study was to ascertain the production of oxidant products (reactive oxidant species (ROS) and NO) and cytokines (TNF- $\alpha$ and IL-6) by rat peritoneal macrophages after a 7-day cocoa diet. To achieve this objective, over a period of 7 days and by oral gavage, female Wistar rats were administered with either water (reference) or cocoa suspensions at doses of $2.4 \mathrm{or} 4.8 \mathrm{~g} / \mathrm{kg} / \mathrm{d}$. One day after the last administration, macrophages were obtained, cultured and stimulated by LPS $(1 \mu \mathrm{g} / \mathrm{ml})$. Supernatants were collected at $6 \mathrm{~h}$ (TNF- $\alpha$ ) or at $24 \mathrm{~h}$ (IL-6 and NO). Levels of TNF- $\alpha$ and IL-6 were quantified by ELISA. Stable end product of $\mathrm{NO}, \mathrm{NO}_{2}{ }^{-}$, was quantified by a modification of Griess reaction. In order to determine ROS production, macrophages were incubated with reduced $2^{\prime}, 7^{\prime}$-dichlorofluorescein diacetate $\left(\mathrm{H}_{2} \mathrm{DCF}-\mathrm{DA}\right)$ for $30 \mathrm{~min}$ at $37^{\circ} \mathrm{C}$. Fluorescence of DCF oxidised by ROS was measured up to $3.5 \mathrm{~h}$. Macrophages from animals administered with $4.8 \mathrm{~g} / \mathrm{kg} / \mathrm{d}$ of cocoa decreased TNF- $\alpha$ secretion by $77 \%$ and $80 \%$ with respect to that from the reference group, under non-stimulation or stimulation conditions, respectively $(P<0.05)$. In addition, a cocoa intake of $4.8 \mathrm{~g} / \mathrm{kg} / \mathrm{d}$ diminished the IL-6 secretion by $94 \%$ in non-stimulated macrophages and $88 \%$ in stimulated ones with respect to the reference cells $(P<0.05)$. No significant differences in TNF- $\alpha$ or IL-6 levels were found in cultures from animals administered with $2.4 \mathrm{~g} / \mathrm{kg}$ cocoa. NO secretion from the $4 \mathrm{v} 8 \mathrm{~g} / \mathrm{kg} / \mathrm{d}$ cocoa group was lower than that quantified in the reference group in any culture condition $(P<0.05)$. ROS production in macrophages from animals receiving $4.8 \mathrm{~g} / \mathrm{kg} / \mathrm{d}$ of cocoa was lower with respect to the reference animals, but it did not reach statistical significance. In summary, a 7-day high cocoa diet reduces the production of oxidants and inflammatory cytokines by peritoneal macrophages ex vivo.

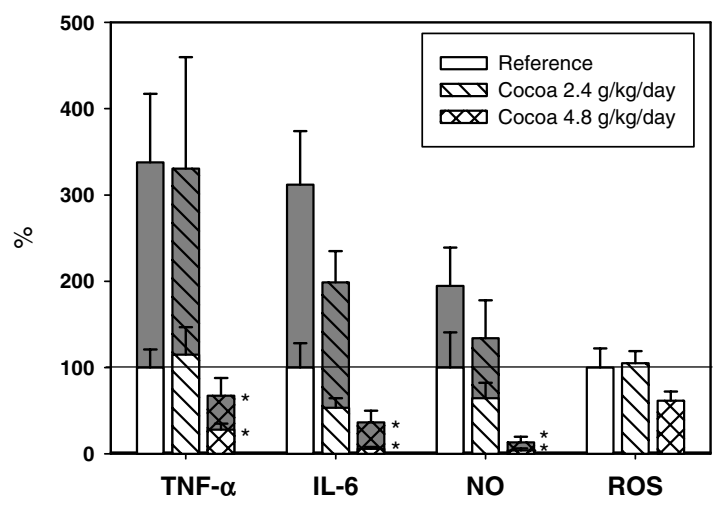

Figure. Relative amount of cytokines and oxidant species produced by peritoneal macrophages. Percentage of TNF- $\alpha$, IL-6, NO or ROS concentrations is expressed with respect to non-stimulated reference values in culture supernatants which was set up to $100 \%$. White bars represent molecule production in non-stimulated cells and each corresponding dark bars represent production in LPS-stimulated cultures. $* P<0.05$ compared with references groups.

This study was supported by the Ministerio de Educación y Ciencia, Spain (AGL2005-002823).

1. Ramiro E, Franch A, Castellote C et al. (2005) J Agric Food Chem 53, 8506-8511.

2. Ono K, Takahashi T, Kamei M et al. (2003) Nutrition 19, 681-685. 